\title{
Balancing a humanoid robot with a prioritized contact force distribution
}

\author{
Alexander Sherikov, Dimitar Dimitrov, Pierre-Brice Wieber \\ INRIA Rhône-Alpes, \\ 38334 Montbonnot Cedex, France \\ E-mail: \{alexander.sherikov, dimitar.dimitrov, pierre-brice.wieber\}@inria.fr
}

\begin{abstract}
Humanoid robots propel themselves and perform tasks by interacting with their environment through contact forces. Typically, nonuniqueness of these forces is dealt with by distributing them evenly between the contacts. In the present paper, we introduce strict prioritization in contact force distribution, to reflect situations when an application of certain contact forces should be avoided as much as possible, for example, due to a fragility of the support. We illustrate this by designing a whole body motion controller for a setting with multiple noncoplanar contacts, where application of an optional contact force is allowed only if it is necessary to maintain balance and execute a task. Balance preservation is addressed by imposing a capturability constraint based on anticipation with a linear model adapted to multiple noncoplanar contacts. The controller is evaluated in simulations.
\end{abstract}

\section{INTRODUCTION}

Humanoid robots belong to the class of floating base systems, and, consequently, the contacts with the environment play a crucial role in their control. The interaction with the environment can be studied by focusing on different aspects such as force distribution between contacts, balance preservation, contact planning for realization of locomotion and manipulation tasks. In the present paper we primarily address the first two aspects in a setting with multiple noncoplanar contacts.

Let us consider the problem of force distribution. In most settings with multiple contacts there exists an infinite number of force distributions that achieve the same base motion. The typical approach to resolve this ambiguity is to make contacts as robust as possible, by keeping each contact force far from the bounds of the respective friction cone, and distribute the forces evenly between all the contacts [1], [2], [3], [4].

There are situations however, such as when a contact area is fragile, when it is preferable to avoid using it unless strictly necessary for balance. In this case, distributing forces evenly between all possible contacts should be avoided. We propose therefore to introduce a prioritized distribution of the contact forces, with the help of hierarchical optimization [1], [5], [6]. We demonstrate our idea in the following setting: a humanoid robot has to reach a target with a hand while standing, it can optionally exploit a contact of the other hand with an additional support to maintain balance. The control goal can be expressed with the informal hierarchy
1: maintain balance

2: reach the target

3: minimize optional contact force

which allows the application of an optional contact force, but only if it is necessary to accomplish the reaching task and maintain balance.

The highest priority in Hierarchy 1 is to preserve balance, i.e., avoid falling. A standard approach for achieving this is to maintain an adequate configuration of the body or position of the Center of Mass (CoM) [1], [2], [4], [7], [8], but this restricts the motions of the robot. A less restrictive alternative is to constrain the CoM to project vertically inside the support area [5], [9]. However, this is valid only for quasi-static motions. In the case of dynamic motions, balance typically requires to use some form of anticipation [10], [11], [12], [13], [14], [15], [16].

Though anticipation using a whole body model is possible [14], [15], [17], it necessitates the use of nonlinear optimization, which can be computationally expensive and is still in the process of being extended to hierarchies [17]. For these reasons we resort to anticipation with a simplified linear model. Note that such models are widely adopted precisely because they are usually sufficient for ensuring balance preservation [10], [11], [12], [18], [19]. In the case of multiple noncoplanar contacts, the simplified models typically involve the contact forces, but neglect the robot's structure and tasks associated with it, such as the reaching task in Hierarchy 1 [11], [12], [13]. As a result, the Hierarchy 1 needs to account for two models at the same time, a whole body model for the reaching task, and a simplified model for balance preservation. A similar approach has already been used in [10] in the case of walking on a flat ground. It can be seen as a whole body Model Predictive Control (MPC), where the whole body model is replaced by a simplified linear model everywhere except the current time instance.

In the present work, we develop an online whole body motion controller with contact prioritization in accordance with Hierarchy 1. The controller is similar to the one presented in [10], but employs a linear model designed to deal with multiple noncoplanar contacts [11], [12]. We construct a capturability constraint to be imposed at the end of the preview. This is important for balance preservation, as recognized in [10], [8], [13] and demonstrated here in simulation. The controller assumes that the contact positions 
are given by an offline planner such as [14], [20]. Since the exact time, when the optional contact is used, is not known in advance, we also assume that the support hand is already at the contact position from the beginning.

The paper is organized as follows. The components of the controller are described in Section II, which focuses on inverse dynamics control, and Section III, where the balance preservation is discussed. The following Section IV presents formalized Hierarchy 1 using the designed controller. Its performance is verified in simulations described in Section V. The final Section VI concludes the paper.

\section{STANDARD WHOLE BODY MOTION CONTROL}

This section briefly describes constraints and objectives used in the standard inverse dynamics approach to control of humanoid robots [21].

\section{A. Lagrangian dynamics}

The dynamics of a humanoid robot having $M$ contacts with the environment can be described as

$$
\underbrace{\left[\begin{array}{l}
\boldsymbol{H}_{1} \\
\boldsymbol{H}_{2} \\
\boldsymbol{H}_{3}
\end{array}\right]}_{\boldsymbol{H}}(\underbrace{\left[\begin{array}{c}
\ddot{\boldsymbol{q}}^{\prime} \\
\ddot{\boldsymbol{x}} \\
\ddot{\boldsymbol{\theta}}
\end{array}\right]}_{\ddot{\boldsymbol{q}}}-\left[\begin{array}{l}
\mathbf{0} \\
\boldsymbol{g} \\
\mathbf{0}
\end{array}\right])+\underbrace{\left[\begin{array}{l}
\boldsymbol{h}_{1} \\
\boldsymbol{h}_{2} \\
\boldsymbol{h}_{3}
\end{array}\right]}_{\boldsymbol{h}}=\left[\begin{array}{l}
\boldsymbol{\tau} \\
\mathbf{0} \\
\mathbf{0}
\end{array}\right]+\underbrace{\left[\begin{array}{l}
\boldsymbol{J}_{c, 1}^{T} \\
\boldsymbol{J}_{c, 2}^{T} \\
\boldsymbol{J}_{c, 3}^{T}
\end{array}\right]}_{\boldsymbol{J}_{c}^{T}} \boldsymbol{f}
$$

where $\boldsymbol{H}$ is the generalized inertia matrix; $\boldsymbol{q}$ is the vector of joint angles $\boldsymbol{q}^{\prime}$, position $\boldsymbol{x}$ of the base of the robot, and Euler angles $\boldsymbol{\theta}$ representing orientation of the base; $\boldsymbol{g}$ is the vector of gravitational acceleration; $\boldsymbol{h}$ is the vector of Coriolis and centrifugal effects; $\boldsymbol{\tau}$ is the vector of joint torques; $f$ is the vector of contact forces $\boldsymbol{f}_{i}$ and moments $\boldsymbol{\mu}_{i}(i=1, \ldots, M)$; and $\boldsymbol{J}_{c}$ is the corresponding Jacobian matrix.

\section{B. Joint limits}

Joint accelerations and torques cannot take arbitrary values due to hardware limitations and therefore must be constrained. We bound the joint torques and accelerations using

$$
\underline{\boldsymbol{\tau}} \leq \boldsymbol{\tau} \leq \overline{\boldsymbol{\tau}}, \quad \ddot{\boldsymbol{q}}^{\prime} \leq \ddot{\boldsymbol{q}}^{\prime} \leq \overline{\ddot{\boldsymbol{q}}}^{\prime}
$$

where the lower and upper bounds $\ddot{\boldsymbol{q}}^{\prime}$ and $\overline{\ddot{q}}^{\prime}$ are constructed to reflect joint position, velocity, and acceleration limits all together [22].

\section{Contact constraints}

Control inputs consistent with the dynamics (1) and respecting hardware limitations may still cause a slip or a loss of contact with the environment, which can be prevented using additional constraints [21]. In these constraints, we denote the contact forces and moments as $\hat{\boldsymbol{f}}_{i}$ and $\hat{\boldsymbol{\mu}}_{i}$ when they are expressed in the frames fixed to the contact points $\boldsymbol{p}_{i}$, with the $z$ axes normal to the contact surfaces.

- The contact forces are subject to friction cone constraints expressed using a linear approximation of the Coulomb friction cone

$$
\boldsymbol{G}_{i} \hat{\boldsymbol{f}}_{i} \leq \mathbf{0}
$$

where the rows of $\boldsymbol{G}_{i}$ are the normal vectors to the edges of the pyramid approximating the friction cone.

- The moment about the $z$ axis is limited due to torsional friction with

$$
\left\|\hat{\boldsymbol{\mu}}_{i}^{z}\right\| \leq \gamma \hat{\boldsymbol{f}}_{i}^{z}
$$

where $\gamma$ is a torsional friction coefficient ([23], Section 5.5).

- The moments about $x$ and $y$ axes cannot be arbitrary as well. The ratio between them and the normal force $\hat{\boldsymbol{f}}_{i}^{z}$ defines the Center of Pressure (CoP), which must stay within the contact support area. Using a linear approximation of the support area boundary, this constraint can be expressed as

$$
\boldsymbol{D}_{i}\left[\begin{array}{c}
\hat{\boldsymbol{f}}_{i} \\
\hat{\boldsymbol{\mu}}_{i}
\end{array}\right] \leq \mathbf{0} .
$$

where $\boldsymbol{D}_{i}$ contains coefficients of the bounding lines [21], [11].

- In order to ensure that the contacts do not slip or detach, the accelerations of contact points are set to zero:

$$
\boldsymbol{J}_{c} \ddot{\boldsymbol{q}}+\dot{\boldsymbol{J}}_{c} \dot{\boldsymbol{q}}=\mathbf{0} .
$$

\section{Control objectives}

We use two simple control objectives throughout this paper. The first one drives a hand to a desired position with the help of a PD controller

$$
\boldsymbol{J}_{r h} \ddot{\boldsymbol{q}}=\Lambda_{p}\left(\boldsymbol{x}_{\text {target }}-\boldsymbol{x}\right)-\Lambda_{d} \dot{\boldsymbol{x}}-\dot{\boldsymbol{J}}_{r h} \dot{\boldsymbol{q}},
$$

where $\Lambda_{d}$ and $\Lambda_{p}$ are positive scalar gains, $\boldsymbol{x}$ and $\dot{\boldsymbol{x}}$ are the the current position and velocity of the end-effector, and $\boldsymbol{x}_{\text {target }}$ is its target position. The second one maintains a reference configuration $\boldsymbol{q}_{\text {ref }}$ of the robot with a joint PDcontroller

$$
\ddot{\boldsymbol{q}}=K_{p}\left(\boldsymbol{q}_{r e f}-\boldsymbol{q}\right)-K_{d} \dot{\boldsymbol{q}},
$$

where $K_{d}$ and $K_{p}$ are positive scalar gains.

\section{BALANCE PRESERVATION}

Similarly to [18], [19] we analyze the problem of balance preservation in terms of viability theory [24]. A state is defined as viable, if there exists at least one evolution starting from this state and not resulting in a fall. In practice, however, most states of interest, when walking, are 2- or 3 - step capturable, what means that the robot is able to stop in 2 or 3 steps [19], [25]. Based on this observation, we already proposed to preview the future motion of the robot, and impose that it must be able to stop some time (corresponding to 2 or 3 steps) in the future [10], i.e., be able to reach a statically balanced state. Note that, similarly to the terminal constraints, which are important components of MPC schemes [26], we impose that the robot stops at a time in the future which is continuously postponed. As a result, it is not imposed that the robot actually stops, only that it maintains the capacity to stop, i.e., capturability.

Motion preview with the whole body dynamical model is a computationally expensive problem, and a simplified model can be used instead. In [10] we used a linear model tailored 
for walking on a flat ground. This same model, however, cannot be employed in a setting with multiple noncoplanar contacts. That is why here we rely on a different model derived from the relationship between the momenta of the robot and the contact forces [11], [12], [14].

\section{A. Preview of momenta of the robot}

The lower parts of the dynamics (1) are the Newton and Euler equations of the whole system [27], involving the linear ${ }^{r} \mathcal{P}$ and angular ${ }^{r} \mathcal{L}$ momenta of the whole robot with respect to the chosen base reference point $r$

$$
\left[\begin{array}{c}
{ }^{r} \dot{\mathcal{P}} \\
\boldsymbol{T}_{\mathcal{E}}^{T}{ }^{r} \dot{\mathcal{L}}
\end{array}\right]=\left[\begin{array}{l}
\boldsymbol{H}_{2} \\
\boldsymbol{H}_{3}
\end{array}\right] \ddot{\boldsymbol{q}}+\left[\begin{array}{l}
\boldsymbol{h}_{2} \\
\boldsymbol{h}_{3}
\end{array}\right]=\left[\begin{array}{l}
\boldsymbol{J}_{c, 2}^{T} \\
\boldsymbol{J}_{c, 3}^{T}
\end{array}\right] \boldsymbol{f}+\left[\begin{array}{l}
\boldsymbol{H}_{2} \\
\boldsymbol{H}_{3}
\end{array}\right]\left[\begin{array}{l}
\mathbf{0} \\
\boldsymbol{g} \\
\mathbf{0}
\end{array}\right]
$$

where $\boldsymbol{T}_{\mathcal{E}}$ is a matrix transforming derivatives of the Euler angles used to represent orientation of the base to the angular velocities. It is more convenient to work with momenta $\mathcal{P}$ and $\mathcal{L}$ expressed in a frame fixed to the CoM [28]. By performing the necessary transformations of (9) we obtain

$$
\left[\begin{array}{c}
\dot{\mathcal{P}} \\
\dot{\mathcal{L}}
\end{array}\right]=\sum_{i=1}^{M}\left[\begin{array}{cc}
\boldsymbol{I} & \mathbf{0} \\
\left(\boldsymbol{p}_{i}-\boldsymbol{c}\right)^{\times} & \boldsymbol{I}
\end{array}\right]\left[\begin{array}{c}
\boldsymbol{f}_{i} \\
\boldsymbol{\mu}_{i}
\end{array}\right]+\left[\begin{array}{c}
m \boldsymbol{g} \\
\mathbf{0}
\end{array}\right]
$$

where $\boldsymbol{I}$ is an identity matrix, $(\cdot)^{\times}$denotes the skewsymmetric matrix corresponding to vector cross product, $\boldsymbol{c}$ is the position of the CoM, $m$ is the mass of the robot. As before, $M$ is the number of contacts and $\boldsymbol{p}_{i}$ are their positions.

Equation (10) can be readily used for motion planning [14], but its nonlinearity entails relatively high computational cost. Instead, we employ a linear model with the following assumptions [11], [12]:

- the CoM is constrained to a horizontal plane

$$
\sum_{i=1}^{M} \boldsymbol{f}_{i}^{z}=m g
$$

provided that the gravitational acceleration is aligned with the $z$ axis, i.e., $\boldsymbol{g}=(0,0,-g)$;

- the equation for the rate of angular momentum about the $z$ axis can be safely neglected.

The evolution of the momenta over $N$ sampling intervals can be previewed using a discrete-time form of this model:

$$
\left[\begin{array}{l}
\tilde{\mathcal{P}}_{k+1}^{x y} \\
\mathcal{P}_{k+1}^{x y} \\
\mathcal{L}_{k+1}^{x y}
\end{array}\right]=\boldsymbol{A}\left[\begin{array}{l}
\tilde{\mathcal{P}}_{k}^{x y} \\
\mathcal{P}_{k}^{x y} \\
\mathcal{L}_{k}^{x y}
\end{array}\right]+\sum_{i=1}^{M_{k}} \boldsymbol{B}_{i, k}\left[\begin{array}{l}
\boldsymbol{f}_{i, k} \\
\boldsymbol{\mu}_{i, k}
\end{array}\right]
$$

where $\tilde{\mathcal{P}}=m \boldsymbol{c}$ is the product of the CoM position by the mass of the robot, superscripts $(\cdot)^{x},(\cdot)^{y}$, and $(\cdot)^{z}$ denote coordinates, and $k=0, \ldots, N-1$. Since the number of contacts and their configurations do not change in our setting, the system is simplified due to $M_{k}=M, \boldsymbol{B}_{i, k}=\boldsymbol{B}_{i}$. The preview starts from the current state $\left(\tilde{\mathcal{P}}_{0}^{x y}, \mathcal{P}_{0}^{x y}, \mathcal{L}_{0}^{x y}\right)$, which can be easily determined from ${ }^{r} \mathcal{P}$ and ${ }^{r} \mathcal{L}$. Control inputs applied on the first sampling interval are the contact forces and moments participating in the whole body dynamics (1), i.e., $\boldsymbol{f}=\left(\boldsymbol{f}_{1,0}, \boldsymbol{\mu}_{1,0}, \ldots, \boldsymbol{f}_{M, 0}, \boldsymbol{\mu}_{M, 0}\right)$. The control inputs remain constant during each sampling interval in the preview, and they must comply with constraints (3), (4), (5), as well as (11). In the end of preview horizon the final state $\left(\tilde{\mathcal{P}}_{N}^{x y}, \mathcal{P}_{N}^{x y}, \mathcal{L}_{N}^{x y}\right)$ and controls $\left(\boldsymbol{f}_{i, N-1}^{x y}, \boldsymbol{\mu}_{i, N-1}^{x y}\right)$ are subject to static balance constraints, which are presented in the next subsection.

\section{B. Static balance constraints}

The key idea of our balance preservation approach is to ensure that commands computed at each control sampling time allow the robot to stop, i.e., reach a statically balanced state. For this purpose we impose the following static balance constraints on the final previewed state and controls of the linear model presented in the previous subsection

- the CoM velocity is zero in the $x-y$ plane, i.e., the linear momentum is zero

$$
\mathcal{P}_{N}^{x y}=\mathbf{0}
$$

- the CoM acceleration is zero in the $x-y$ plane, i.e., the total force applied to the CoM is zero:

$$
\sum_{i=1}^{M} \boldsymbol{f}_{i, N-1}^{x y}=\mathbf{0}
$$

- the angular momentum is zero about $x$ and $y$ axes:

$$
\mathcal{L}_{N}^{x y}=\mathbf{0}
$$

- the rate of change of the angular momentum about $x$ and $y$ axes is also zero:

$$
\left[\begin{array}{l}
\tilde{\mathcal{P}}_{N}^{x} g-\sum_{i=1}^{M}\left(\boldsymbol{f}_{i, N-1}^{z} \boldsymbol{p}_{i}^{x}-\boldsymbol{\mu}_{i, N-1}^{y}\right) \\
\tilde{\mathcal{P}}_{N}^{y} g-\sum_{i=1}^{M}\left(\boldsymbol{f}_{i, N-1}^{z} \boldsymbol{p}_{i}^{y}+\boldsymbol{\mu}_{i, N-1}^{x}\right)
\end{array}\right]=\mathbf{0} .
$$

IV. A HIERARCHY WITH FORCE PRIORITIZATION

Given all the components described in Sections II and III, we can formalize Hierarchy 1 as follows:

1: Equality constraints

$$
\text { Hierarchy (2) }
$$

- dynamics (1)

- fixed contacts (6)

- zero vertical acceleration (11)

2: Inequality constraints

- joint limits (2)

- contact constraints (3), (4), (5)

3: Static balance constraints (14), (13), (15), (16)

4: Reaching task (7)

5: Minimization of the optional contact force

6: Final objectives

- Maintaining the reference configuration using (8), weighted with the inertia matrix $\boldsymbol{H}$

- Minimization of all contact wrenches

Decision variables of this optimization problem are the joint torques $\tau$, the generalized accelerations $\ddot{\boldsymbol{q}}$, and contact wrenches $\left(\boldsymbol{f}_{i, k}, \boldsymbol{\mu}_{i, k}\right)$ for each $i$-th contact and $k$-th sampling interval of the preview horizon. The joint torques are eliminated from computations as in [3]. The exact definition of 


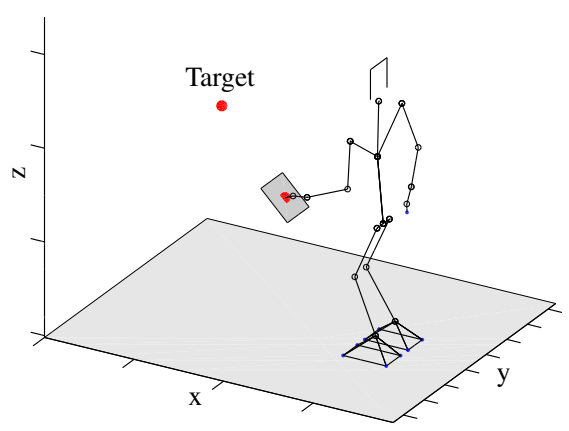

(a) initial

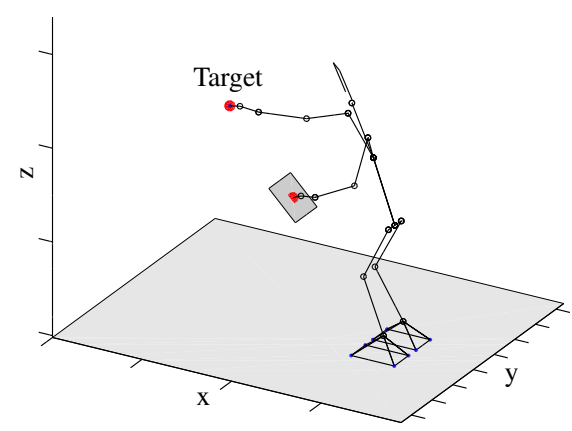

(b) after 15 seconds

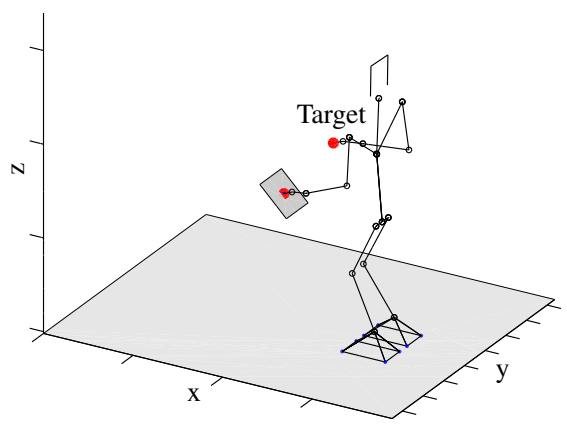

(c) after 25 seconds

Fig. 1: Test 2: configurations of the robot during the simulation. Grey areas represent contact surfaces.

the objective to minimize on the 5-th level concerning the optional contact force is left for Section V.

\section{Simulations}

We evaluate Hierarchy 2 in simulations using the HRP-2 robot [29], whose behavior can be examined in the accompanying video.

All tests presented in this section are performed in the same general setting: the robot is standing with its left hand positioned on an additional support, while the right hand either has to move to a given target position (Figure 1) or maintain its position in presence of disturbances (Figure 6). When the goal is to reach a target position, the target is initially set to be far from the robot and then moved closer as demonstrated in Figures 1 and 2. In all cases the controller is expected to apply a force on the additional support only if necessary to preserve balance.

We have observed that particular choices of the preview horizon length and its discretization have little effect on the overall behavior of the robot across all our tests. In all the following tests the MPC parameters are set as follows: the number of sampling intervals in the preview $N=4$, with a duration of one interval $T=100 \mathrm{~ms}$.

In order to successfully complete these tests, it is necessary to regularize Hierarchy 2 in the presence of conflicts between its levels. Otherwise, the joint accelerations start to flip between the bounds and the right hand task is poorly executed. We perform regularization of the levels 4 and 5, which may conflict with each other and the balance constraints, using the weighted objective from the last level 6 .

\section{A. Test 1}

Here, the right hand has to reach a moving target position, as demonstrated in Figures 1 and 2. Before this can be achieved, Hierarchy 2 has to be completed by defining its 5-th level. One possible way to do this is to perform a minimization of the current contact force, using

$$
\boldsymbol{f}_{\text {opt }, 0}=\mathbf{0} .
$$

However, a controller based on such formulation does not produce the desired behavior since it does not use its knowledge of the future adequately.
First of all, the inequality constraints on the level 2 become infeasible during the simulation. The reason for this is that the previewed optional contact forces can take large values, which cannot be applied by the robot. We address this problem by introducing upper bounds on the normal components of the previewed optional contact forces

$$
\boldsymbol{f}_{o p t, k}^{\perp} \leq \bar{f}_{\max }, \quad k=0, \ldots, N-1
$$

on the 2-nd level of Hierarchy 2. This helps to reflect the hand joint limitations, which cannot be directly expressed in the preview. Though the proposed modification helps to avoid infeasibilities, it is still not sufficient to obtain the desired behavior: the increase of the contact force is very sharp (Figure 3) and the CoM (Figure 5) does not return close to the initial position after the hand target is moved back.

The sharp increase of the contact force occurs due to the fact that the objective (17) favors large future contact forces $\boldsymbol{f}_{\text {opt }, k}(k=1, \ldots, N-1)$ in order to minimize the current force $f_{\text {opt }, 0}$. We can see in Figures 3 and 5 that once the CoM leaves the support area of the feet, the robot starts falling forward until it is absolutely necessary to apply the current optional contact force $\boldsymbol{f}_{o p t, 0}$ to stop falling.

In the second part of the motion the objective (17) prevents the controller from momentarily increasing the current optional contact force $f_{o p t, 0}$, what is necessary to move the CoM back towards the initial state.

\section{B. Test 2}

In order to address the problems indicated in Test 1 , we propose to minimize the optional contact force over the whole preview horizon:

$$
\boldsymbol{f}_{o p t, k}=\mathbf{0}, \quad k=0, \ldots, N-1 .
$$

By keeping the previewed optional contact forces small, we remove the need for additional feasibility constraints (18) in the considered setting. Furthermore, the objective (19) allows a larger current contact force if it helps to decrease the contact forces in the future.

The obtained controller works completely in accordance with our expectations (see Figure 1). Initially, the optional contact force is zero and starts increasing at second 5 of the simulation until the target is reached (see Figures 3,2), after 


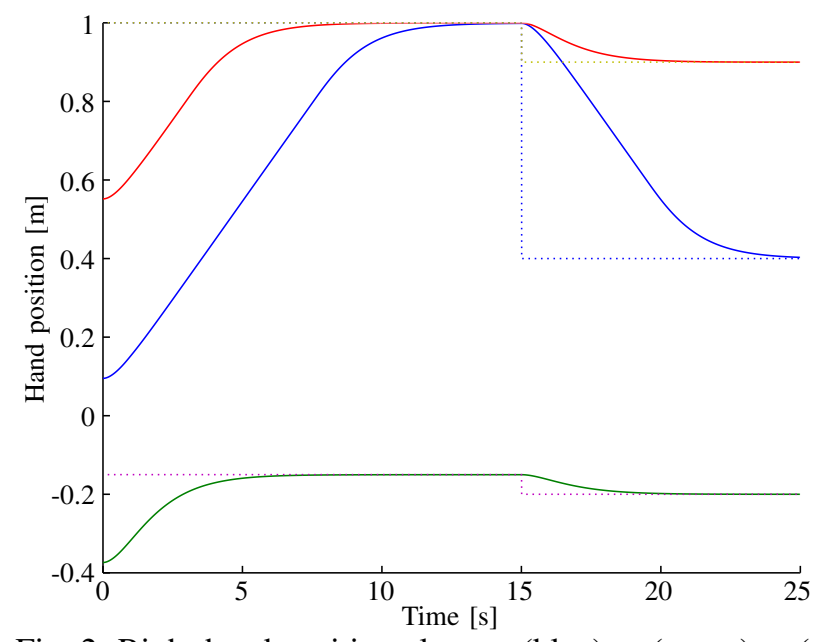

Fig. 2: Right hand position along $x$ (blue), $y$ (green), $z$ (red) axes. The desired positions are shown in dotted lines. Solid curves indicate trajectories executed in test 2 .

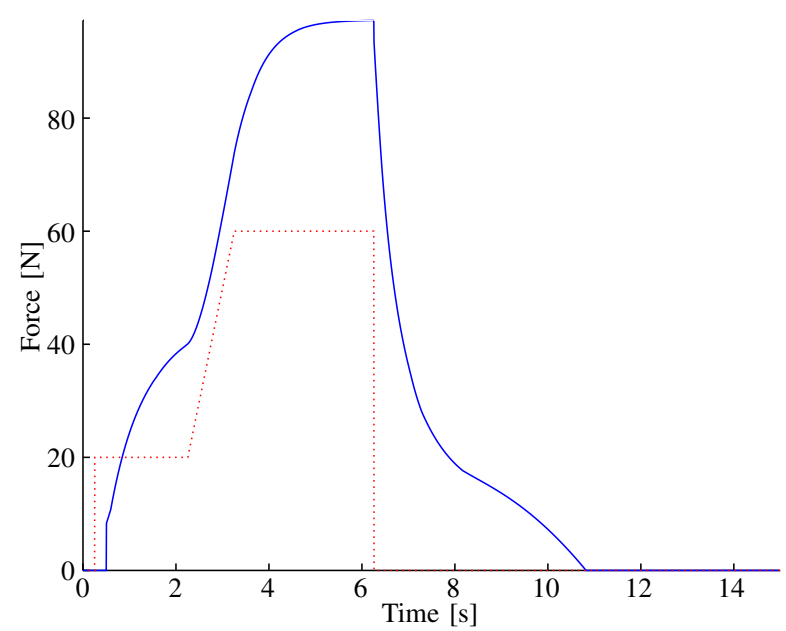

Fig. 4: Norms of the optional contact force $\boldsymbol{f}_{\text {opt }, 0}$ (solid blue) and the external force $\boldsymbol{f}_{\text {ext }}$ (dotted red) in test 3 .

the change in position of the target the force gradually returns to zero. Note that contrary to the Test 1 , the moment of the contact activation is very close to the moment when the CoM projection leaves the foot support area, as demonstrated in Figure 5. Thus, the optional contact force is applied before it is strictly necessary thanks to the anticipation done in the objective (19).

\section{Test 3}

The controller used in Test 2 also produces a satisfying behavior in the presence of perturbations. We verify this in a setting where the right hand has to maintain a constant position under action of an external force $\boldsymbol{f}_{\text {ext }}$, as shown in Figure 6. Thus, the goal of the controller is to maintain balance while holding a heavy object, such as a bucket filled with some weight [30]. The external force is assumed to be measured and its contribution is accounted for in the dynamical model (1), but not in the preview.

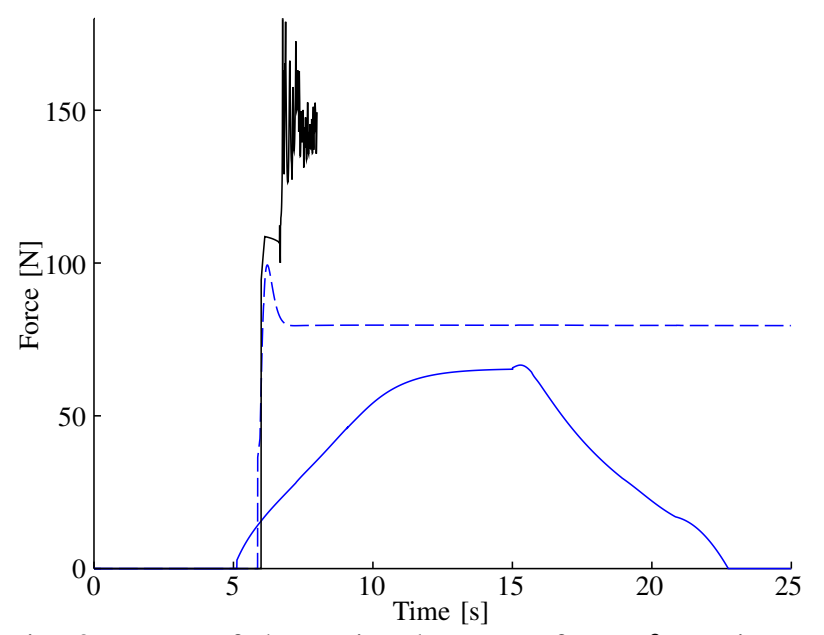

Fig. 3: Norm of the optional contact force $\boldsymbol{f}_{\text {opt }, 0}$ in test 1 (dashed blue), test 2 (solid blue), test 4 (solid black).

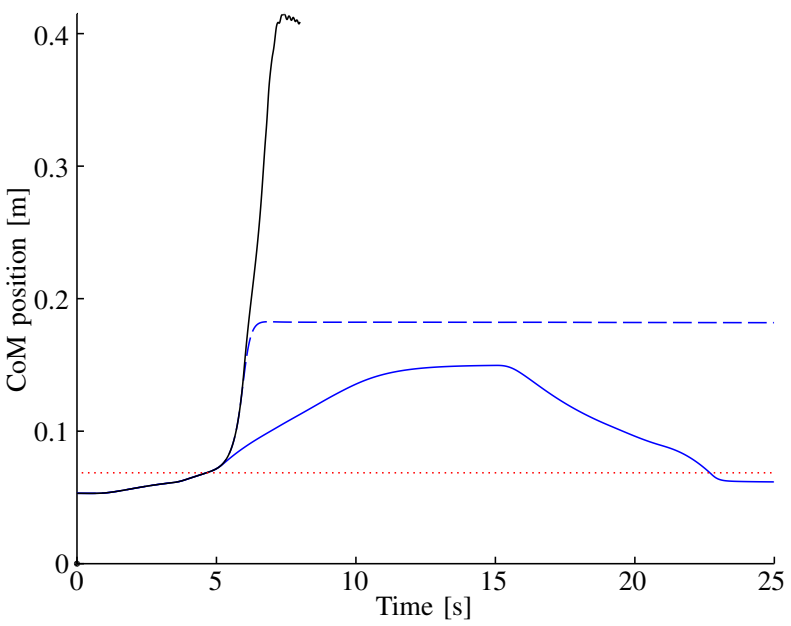

Fig. 5: Position of the CoM along the $x$ axis in test 1 (dashed blue), test 2 (solid blue), test 4 (solid black), and the bounds of the foot support area (dotted red).

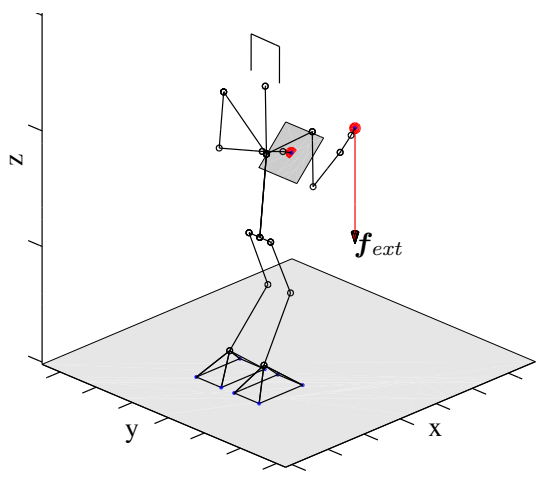

Fig. 6: Initial configuration of the robot in test 3 .

The evolution of the optional contact force and the external force are shown in Figure 4. The external force of $20 \mathrm{~N}$ is initially applied at $0.25 \mathrm{~s}, 2$ seconds later it starts to grow during 1 second until it reaches $60 \mathrm{~N}$, and is set back to 0 
at $6.25 \mathrm{~s}$. The optional contact force changes smoothly in response to the disturbance, and eventually returns to 0 .

\section{Test 4}

Finally, Test 2 was repeated without the static balance constraints (level 3 of Hierarchy 2), to demonstrate their importance for balance preservation. During the simulation the robot falls towards the target as can be seen in Figures 3, 5 eventually causing a major violation of the constraints.

\section{CONCLUSION}

We designed a whole body motion controller with contact force prioritization and balance constraints for general multicontact scenarios. The controller was evaluated in simulations, which demonstrated that it is capable of applying an optional contact force only when necessary to execute tasks and maintain balance. Furthermore, we indicated that some minor design choices can lead to unsatisfactory behavior, and additional constraints on the previewed motion may be necessary to reflect the whole body limitations.

\section{ACKNOWLEDGMENTS}

This work has been funded by the PSPC Romeo 2 project and EU H2020 Comanoid Research and Innovation Action (RIA).

\section{REFERENCES}

[1] L. Saab, O. E. Ramos, F. Keith, N. Mansard, P. Souères, and J.-Y. Fourquet, "Dynamic whole-body motion generation under rigid contacts and other unilateral constraints," IEEE Transactions on Robotics, vol. 29, no. 2, pp. 346-362, 2013.

[2] C. Ott, M. Roa, and G. Hirzinger, "Posture and balance control for biped robots based on contact force optimization," in Humanoid Robots (Humanoids), 2011 11th IEEE-RAS International Conference on, Oct 2011, pp. 26-33.

[3] A. Herzog, L. Righetti, F. Grimminger, P. Pastor, and S. Schaal, "Balancing experiments on a torque-controlled humanoid with hierarchical inverse dynamics," in Intelligent Robots and Systems (IROS 2014), 2014 IEEE/RSJ International Conference on, Sept 2014, pp. 981-988.

[4] S. Hyon, J. Hale, and G. Cheng, "Full-body compliant human humanoid interaction: Balancing in the presence of unknown external forces," Robotics, IEEE Transactions on, vol. 23, no. 5, pp. 884-898, Oct 2007.

[5] A. Escande, N. Mansard, and P.-B. Wieber, "Hierarchical quadratic programming: Fast online humanoid-robot motion generation," The International Journal of Robotics Research, 2014.

[6] O. Kanoun, F. Lamiraux, and P.-B. Wieber, "Kinematic control of redundant manipulators: Generalizing the task-priority framework to inequality task," Robotics, IEEE Transactions on, vol. 27, no. 4, pp. 785-792, Aug 2011.

[7] C. Collette, A. Micaelli, C. Andriot, and P. Lemerle, "Robust balance optimization control of humanoid robots with multiple non coplanar grasps and frictional contacts," in Robotics and Automation, 2008. ICRA 2008. IEEE International Conference on, May 2008, pp. 31873193.

[8] B. Henze, C. Ott, and M. Roa, "Posture and balance control for humanoid robots in multi-contact scenarios based on model predictive control," in Intelligent Robots and Systems (IROS 2014), 2014 IEEE/RSJ International Conference on, Sept 2014, pp. 3253-3258.

[9] T. Bretl and S. Lall, "Testing static equilibrium for legged robots," Robotics, IEEE Transactions on, vol. 24, no. 4, pp. 794-807, Aug 2008.

[10] A. Sherikov, D. Dimitrov, and P.-B. Wieber, "Whole body motion controller with long-term balance constraints," in Humanoid Robots (Humanoids), 2014 14th IEEE-RAS International Conference on, Nov 2014, pp. 444-450.
[11] K. Nagasaka, T. Fukushima, and H. Shimomura, "Whole-body control of a humanoid robot based on generalized inverse dynamics and multicontact stabilizer that can take account of contact constraints," in the Proceedings of the 17th Robotics Symposia, 2012, pp. 134-141.

[12] H. Audren, J. Vaillant, A. Kheddar, A. Escande, K. Kaneko, and E. Yoshida, "Model preview control in multi-contact motionapplication to a humanoid robot," in Intelligent Robots and Systems (IROS 2014), 2014 IEEE/RSJ International Conference on, Sept 2014, pp. 4030-4035.

[13] D. Mansour, A. Micaelli, A. Escande, and P. Lemerle, "A new optimization based approach for push recovery in case of multiple noncoplanar contacts," in Humanoid Robots (Humanoids), 2011 11th IEEE-RAS International Conference on, Oct 2011, pp. 331-338.

[14] H. Dai, A. Valenzuela, and R. Tedrake, "Whole-body motion planning with centroidal dynamics and full kinematics," in Humanoid Robots (Humanoids), 2014 14th IEEE-RAS International Conference on, Nov 2014, pp. 295-302.

[15] Y. Tassa, T. Erez, and E. Todorov, "Synthesis and stabilization of complex behaviors through online trajectory optimization," in Intelligent Robots and Systems (IROS), 2012 IEEE/RSJ International Conference on, Oct 2012, pp. 4906-4913.

[16] L. Sentis, J. Park, and O. Khatib, "Compliant control of multicontact and center-of-mass behaviors in humanoid robots," Robotics, IEEE Transactions on, vol. 26, no. 3, pp. 483-501, June 2010.

[17] F. Romano, A. Del Prete, N. Mansard, and F. Nori, "Prioritized Optimal Control: a Hierarchical Differential Dynamic Programming approach," in Robotics and Automation (ICRA), 2015 IEEE International Conference on, 2015.

[18] P.-B. Wieber, "Viability and Predictive Control for Safe Locomotion," in IEEE-RSJ International Conference on Intelligent Robots \& Systems, Nice, France, 2008.

[19] T. Koolen, T. de Boer, J. Rebula, A. Goswami, and J. Pratt, "Capturability-based analysis and control of legged locomotion, part 1: Theory and application to three simple gait models," The International Journal of Robotics Research, vol. 31, no. 9, pp. 1094-1113, 2012.

[20] K. Bouyarmane, A. Escande, F. Lamiraux, and A. Kheddar, "Potential field guide for humanoid multicontacts acyclic motion planning," in Robotics and Automation, 2009. ICRA '09. IEEE International Conference on, May 2009, pp. 1165-1170.

[21] Y. Fujimoto and A. Kawamura, "Proposal of biped walking control based on robust hybrid position/force control," in Robotics and Automation, 1996. Proceedings., 1996 IEEE International Conference on, vol. 3, Apr 1996, pp. 2724-2730 vol.3.

[22] S. Rubrecht, V. Padois, P. Bidaud, M. de Broissia, and M. Da Silva Simoes, "Motion safety and constraints compatibility for multibody robots," Autonomous Robots, vol. 32, no. 3, pp. 333-349, 2012.

[23] R. Murray, Z. Li, and S. Sastry, A Mathematical Introduction to Robotic Manipulation. Taylor \& Francis, 1994.

[24] J.-P. Aubin, A. Bayen, and P. Saint-Pierre, Viability Theory: New Directions. Springer, 2011.

[25] P. Zaytsev, S. J. Hasaneini, and A. Ruina, "Two steps is enough: no need to plan far ahead for walking balance," in Proceedings of the International Conference on Robotics and Automation (ICRA), May 2015.

[26] D. Mayne, J. Rawlings, C. Rao, and P. Scokaert, "Constrained model predictive control: Stability and optimality," Automatica, vol. 36, no. 6, pp. $789-814,2000$.

[27] P.-B. Wieber, "Holonomy and nonholonomy in the dynamics of articulated motion," in Fast Motions in Biomechanics and Robotics, ser. Lecture Notes in Control and Information Sciences, M. Diehl and K. Mombaur, Eds. Springer Berlin Heidelberg, 2006, vol. 340, pp. 411-425.

[28] D. Orin, A. Goswami, and S.-H. Lee, "Centroidal dynamics of a humanoid robot," Autonomous Robots, vol. 35, no. 2-3, pp. 161-176, 2013.

[29] K. Kaneko, F. Kanehiro, S. Kajita, H. Hirukawa, T. Kawasaki, M. Hirata, K. Akachi, and T. Isozumi, "Humanoid robot hrp-2," in Robotics and Automation, 2004. Proceedings. ICRA '04. 2004 IEEE International Conference on, vol. 2, April 2004, pp. 1083-1090 Vol.2.

[30] B. Stephens and C. Atkeson, "Dynamic balance force control for compliant humanoid robots," in Intelligent Robots and Systems (IROS), 2010 IEEE/RSJ International Conference on, Oct 2010, pp. 12481255 . 